\title{
Les jeux guerriers du Cameroun de l'Ouest. Quelques propos iconoclastes
}

Jean-Pierre Warnier

\section{(2) OpenEdition}

1 Journals

Édition électronique

URL : https://journals.openedition.org/tc/188

DOI : $10.4000 /$ tc. 188

ISSN : 1952-420X

Éditeur

Éditions de l'EHESS

\section{Édition imprimée}

Date de publication : 1 juin 2002

ISSN : 0248-6016

\section{Référence électronique}

Jean-Pierre Warnier, "Les jeux guerriers du Cameroun de l'Ouest. Quelques propos iconoclastes », Techniques \& Culture [En ligne], 39 | 2002, mis en ligne le 13 juin 2006, consulté le 29 septembre 2022. URL : http://journals.openedition.org/tc/188; DOI : https://doi.org/10.4000/tc.188

Ce document a été généré automatiquement le 29 septembre 2022.

Tous droits réservés 


\title{
Les jeux guerriers du Cameroun de l'Ouest. Quelques propos iconoclastes
}

\author{
Jean-Pierre Warnier
}

1 Lorsque je l'ai reçu, il m'est apparu que l'appel à contribution lancé par Techniques \& Culture sur les sports et les jeux du corps au sein des sociétés « exotiques " promettait des développements originaux hors des sentiers battus. Pourtant, j'ai hésité à y répondre. Je ne pouvais en effet que tenir des propos iconoclastes par rapport à la problématique proposée. Je cite : «le corps-moteur comme moyen d'action et à la fois comme objet ou matière d'œuvre modelée par l'action». Cela dit, le pluralisme de bon aloi de la revue m'a convaincu de surmonter mes hésitations et de tenir dans ses pages les propos iconoclastes en question.

2 Je procéderai en deux temps, d'abord par des considérations théoriques sur les techniques du corps et la notion de sujet, ensuite par une esquisse ethnographique tirée de mes terrains camerounais.

«Les Techniques du corps » et le sujet

Le fameux texte de Marcel Mauss sur les techniques du corps est égarant et génial. Égarant car la conceptualisation en est insuffisante et maladroite et que le vocabulaire en est, pour des lecteurs du début du XXIe siècle, obsolète. La notion de « corps » qui est celle de Mauss est rudimentaire. C'est le corps anatomo-physiologique. Mauss lisait assidûment les écrits des psychologues de son temps : Dumas, Head, Freud, Janet. Bruno Karsenti (1997) a montré de manière convaincante que, de 1900 à 1921, Mauss s'est considérablement rapproché des psychologues, et qu'il a réussi à formuler la manière dont la sociologie résolvait les apories de la psychologie -et vice-versa. C'est en effet le social qui fait la médiation entre l'intelligence rationnelle et les affects « irrationnels » que les psychologues ne parvenaient pas à articuler entre eux. C'est la psychologie qui nous renseigne sur les "roues d'engrenage » entre le biologique et le social que la sociologie peinait à comprendre. 
4 Ce sont des articles du psychologue Dumas sur le rire et les larmes - phénomènes neurophysiologiques, affectifs et socialement codés (donc «biologiques», "psychologiques » et «sociaux » dans le vocabulaire de Mauss) - qui ont joué le rôle d'amorce de la réflexion maussienne sur les techniques du corps.

5 Mauss avait donc engagé un dialogue fort avec les psychologues. Pourtant, bien qu'il ait fréquenté les écrits de Head, il ignorait ceux de Schilder sur le schéma corporel, dont les premiers datent de 1923 (alors que le texte de Mauss sur les techniques du corps leur est postérieur de treize ans: 1936). L'apport de Schilder (1923 et 1935) est fondamental : ce qu'on appelle « le corps » n'est pas le corps anatomo-physiologique (la " viande ", pourrait-on dire), mais une synthèse sensori-affectivo-motrice construite au fil des ans par apprentissage dans un monde matériel donné. Cette synthèse est modulable à l'infini en fonction des variations de l'environnement et des conduites du sujet. Elle est à géométrie variable et elle intègre tour à tour tous les objets avec lesquels nous faisons corps, selon nos conduites motrices du moment. Cette synthèse est socialement normée. Elle mobilise les affects du sujet.

6 Par conséquent, Mauss ne disposait pas des concepts qui lui auraient permis de comprendre ce qu'il affirmait ne pas comprendre, à savoir comment un Kabyle dévale une pente ou un escalier sans perdre ses babouches ou comment les femmes peuvent marcher avec des chaussures à talons hauts. C'est que ces objets ne sont pas extérieurs au « corps ", comme la notion de « corps-viande » le laisse à penser. Ils sont inclus dans le schéma corporel ou sensori-affectivo-moteur du sujet, comme la canne de l'aveugle ou le chapeau de l'élégante, ainsi que le notait Schilder.

7 L'intuition d'un homme «bio-psycho-social» est égarante quoique géniale, car «le corps » n'est pas réductible à « la viande ». Mais il y a plus. "L'homme total », auquel nos collègues ethnologues font facilement référence, par déférence pour Mauss, est également égarant. Y aurait-il un homme partiel ? et peut-on faire un homme avec un " corps-viande »? Traduit en langage contemporain, c'est en fait du sujet qu'il s'agit. Mais, à l'époque où Mauss écrivait, le terme de «sujet » était préempté par le droit, la médecine, et surtout la philosophie. Il était indisponible pour la sociologie, puisque sa variante philosophique y était pour l'essentiel irrecevable. Le sujet du philosophe, à l'époque, était un sujet avant tout pensant, conscient de soi et du monde, moralement construit, et calé sur le cogito, alors que « l'homme total » selon Mauss était en outre mû par des passions, socialement conditionné, et fondamentalement corporel.

8 Au début du XXIe siècle, nous ne rencontrons plus les mêmes problèmes. Le sujet de l'humanisme, sous ses variétés chrétienne ou athée, est mort sous les coups des sciences de l'homme et de la société comme des événements historiques de ce siècle de fer que fut le XXe siècle (et que promet d'être le XXIe). Le terme de «sujet » devient disponible pour d'autres usages, et c'est précisément «l'homme total» de Mauss, qui en constitue l'ébauche, et dont il faut préciser le portrait à la lumière des recherches de penseurs aussi divers que Schilder, Winnicott, Dolto, Parlebas, Foucault, etc.

9 Ainsi, le Mauss des «Techniques du corps » est égarant. Mais il est génial : il met le doigt, dès les années vingt, sur toutes les insuffisances de notre anthropologie: l'obsession pour le langage, le signe et la communication; l'incapacité à prendre en compte le corps, la culture matérielle, les affects et les passions; la réticence à chercher comment le sujet articule conduites sensori-affectivo-motrices et langage, intériorisation et communication. Mauss trace le programme de ce que devrait être une anthropologie générale fondée sur la notion de sujet, alias « homme total », qui donne 
d'emblée le monisme de ce que les phénoménologues - dont Merleau-Ponty, lecteur assidu de Schilder- appelaient «l'être-au-monde ».

10 Je suis maintenant à pied d'œuvre pour dire pourquoi je suis en délicatesse avec l'appel à communication lancé par Techniques \& Culture. C'est -je cite- que la " problématique des techniques du corps : le corps-moteur comme moyen d'action et à la fois comme objet ou matière d'œuvre modelée par l'action» plaque sur le sujet une conceptualisation et une terminologie mécanistes, objectivantes, dualistes, et plus égarantes encore que celle de Mauss puisqu'elles n'ont pas l'excuse du contexte historique et de la date.

11 Cette problématique est celle de la technologie culturelle qui, à la suite de Mauss, considère la technique comme "une action traditionnelle efficace sur la matière ». C'est donc une action transitive, qui va du «corps » agissant vers la matière ouvrée, afin de la transformer. C'est une action qui se déroule en extériorité, l'ouvrier étant bien distinct de la matière, comme l'est le sujet de l'objet. Cette problématique est solidaire d'une notion de corps pré-schilderienne. Mais, elle prend au pied de la lettre la distinction faite par Mauss entre les «techniques du corps » (sans médiation par les objets matériels) et les "techniques à instruments " (avec médiation, et sans effet présumé sur le sujet), alors même qu'une lecture attentive de Schilder montre que cette distinction est intenable, et que, de facto, Mauss ne parvient pas à s'y tenir : dans « Les techniques du corps ", les objets, chassés par la porte, rentrent par la fenêtre sans pouvoir être analysés (les babouches, les chaussures à talon, mais aussi les bêches des poilus de 1914, les instruments de musique, la literie, le mobilier).

12 En d'autres termes, le technicien est, à mes yeux, un sujet agissant, engagé dans des conduites sensori-affectivo-motrices, si bien que l'action sur la matière est, de manière indissociable, une action de soi sur soi, une technique de soi. Le schéma corporel du sujet se dilate jusqu'à englober, par l'affect, la préhension et la perception sensorimotrice, tout ce qui, dans l'environne-ment, possède quelque pertinence à l'endroit de l'action du sujet. On n'a pas un corps-viande agissant de manière extérieure et transitive sur une matière inerte, y compris la matière de son propre corps (par exemple le bras-muscle massant l'objet mollet). On a un sujet qui fait corps avec les éléments pertinents de son environnement sensori-affectivo-moteur. Cela dans tous les cas, sous tous les cieux, à toutes les époques et dans toutes les sociétés. C'est un universel.

13 Cette proposition n'est ni un postulat ni un axiome. La praxéologie motrice développée par Pierre Parlebas (1999) sur la base d'une masse considé-rable de travaux antérieurs permet d'en établir le bien fondé.

Culture matérielle et subjectivation

14 La proposition précédente possède un corollaire de première importance, à savoir que, d'un point de vue analytique, il n'est pas pertinent de distinguer « la technique » d'une part et « la société » ou « l'acteur » d'autre part. Le paradigme schilderien opère une rupture, parallèle à celle qui fut maladroitement et incomplètement suggérée par Mauss. Cette rupture est ontologique et épistémologique. Elle disqualifie la "dichotomie fatale» entre technique et société qui a tant préoccupé Bruno Latour, Pierre Lemonnier et bien d'autres. Si le sujet de l'histoire est le sujet-et-son-universsensori-moteur, alors, il n'est pas pertinent de chercher des facteurs de changements ou le vecteur de l'histoire en dehors de cette monade en prise avec les autres sujets. 
15 Dans la langue française, je ne vois pas d'autre vocable que celui de «sujet » qui fasse l'affaire et qui désigne ce dont il s'agit. Après la mort du sujet de l'humanisme, ce vocable est disponible pour les sciences de l'hommes et de la société. La question centrale est d'en cerner les contours et d'en fixer plus ou moins le contenu. Je vais donc me permettre une reformulation iconoclaste de la problématique du présent numéro thématique de Techniques \& Culture en me rattachant «à la vaste problématique des techniques de soi : le sujet agissant sur lui-même comme acteur et objet de ses propres actions médiatisées par son environnement matériel et humain ». Dans la liste des thèmes de réflexion proposés par l'appel à contribution, je choisirai « la construction du sujet par les sports et les jeux du corps ».

Les lecteurs de Foucault apprécieront à quel point ces formulations sont proches des siennes et de ce qu'il désigne par le terme de « subjectivation » qui est le processus par lequel on devient un sujet qui apprend à se gouverner, à agir sur soi, tout en étant assujetti à des réseaux d'actions en nombre incalculable par lesquelles les sujets agissent sur les actions des autres de manière plus ou moins récurrente, institutionnalisée et socialement configurée, ce qui entraîne un puissant effet de production d'identité. Foucault est un auteur contesté dans les sciences de l'homme et de la société. Mais s'il s'agit de cerner la notion de sujet, ses travaux offrent des pistes de travail irremplaçables, quitte à apporter des correctifs importants aux éléments théoriques proposés par le philosophe, ce que je ne ferai pas ici, faute de place.

Dans cette perspective, la frontière entre travail, technique, jeu et sports se redessine selon des lignes inédites (même si, du point de vue de ce que P. Parlebas appelle des "logiques internes", ces activités se différencient en plusieurs grandes catégories). Je veux dire qu'il n'existe aucune action efficace sur la matière qui ne soit, indissociablement, une action efficace de soi sur soi. En termes maussiens, pas de technique d'objet qui ne soit une technique de soi. Au demeurant, les travaux de technologie culturelle nous ont appris combien l'activité travailleuse peut identifier le travailleur et le configurer dans ses capacités sensorielles, affectives et motrices.

La présente esquisse ontologico-épistémologique est extrêmement rudimentaire. Pour de plus amples développements, je me permets de renvoyer le lecteur aux publications de Julien et Warnier (1999), Warnier (1999 et 2001), Parlebas (1999), Tisseron (1999 et 2000), et je me tournerai maintenant vers les Grassfields du Cameroun pour une esquisse ethnographique de «la construction du sujet par les jeux du corps».

La construction des guerriers

19 Le rapport entre guerre et sports a déjà fait couler beaucoup d'encre, en provenance des meilleurs encriers, comme celui de Norbert Elias. Dans les chefferies montagnardes du Cameroun de l'ouest (Warnier 1985), la guerre fut une pratique régulière au cours des deux derniers siècles, mais elle n'a donné lieu à aucun processus de sportification. J'examinerai donc les pratiques sensori-motrices autour de la guerre, mais qui ne peuvent être qualifiées ni de jeu ni de sport.

Dans cette région d'Afrique, la définition vernaculaire de la guerre est simple. Désignée en langue ngemba du nom de ntso, elle consiste à se battre avec des armes en fer (javelots, fusils, matchètes) contre des ennemis avec lesquels il n'existe, au niveau politique, ni alliance formelle, ni lien généalogique opératoire. La guerre a pour objectif de tuer des ennemis, de s'emparer de leur crâne, de faire intrusion dans les limites de la chefferie adverse, et d'y saisir des biens inaliénables comme des récipients et statues conservées au palais du chef. D'un point de vue analytique (à quoi ne correspond 
aucune verbalisation par les intéressés), il s'agit de crever les enveloppes de l'ennemi au moyen d'armes tranchantes -que ce soit l'enveloppe des sujets individuels ou celle $\mathrm{du}$ « récipient » qu'est la chefferie adverse-, afin d'en faire échapper la substance ou de se l'approprier.

La guerre (ntso) s'oppose à la rixe (filame) pratiquée avec des armes en bois -et non en fer-contre des ennemis/partenaires avec lesquels on est lié par une alliance politique ou par la parenté. Du point de vue analytique encore, le but est de rosser l'adversaire et d'attirer l'attention des notables et des chefs en vue d'un arbitrage, sans crever les enveloppes des sujets individuels ou des groupes.

La guerre et la rixe sont des actes, au sens où Gérard Mendel (1998) en parle. C'est-àdire qu'elles comportent essentiellement une incertitude et un risque : celui de se faire crever la peau ou de se faire rosser, ou de voir les notables arbitrer en votre défaveur. Le sujet apparaît par conséquent totalement impliqué dans l'un et l'autre de ces deux cas, au double sens foucaldien de sujet : comme sujet de ses propres actions et comme sujet assujetti à une souveraineté politique.

Ayant délimité les contours de la guerre par opposition à la rixe, je laisserai de côté la seconde pour me pencher exclusivement sur la première. Mais je décalerai encore le regard d'un cran, et le ferai porter non sur les actes guerriers (dont, bien entendu, je n'ai pas été le témoin), mais sur les simulations quasi ludiques qu'en donnent les hommes lors des festivals annuels et des assemblées occasionnelles des loges de guerriers appelées manjong.

Ces simulations ont quelque chose de ludique, tout en n'étant pas des jeux, en ce sens qu'elle ne sont pas accomplies "pour de vrai», qu'elle constituent des conduites sensori-motrices impliquant des actions motrices observables, qu'elles procurent du plaisir, qu'elles sont socialisées. Ces jeux ne font l'objet d'aucune «sportification » au sens de Parlebas, à l'inverse du lancer du javelot, du saut d'obstacle ou de l'escrime dans les sociétés occidentales modernes. Les chefferies «traditionnelles» des Grassfields pratiquent le jeu -pas le sport. Voici une description ethnographique succincte de quelques-unes de ces pratiques.

Le jour de mesongong

Lors de Njwi mesongong (le jour de mesongong, une fois l'an, fin décembre), les hommes de la loge guerrière du lignage royal, en tenue guerrière (pagne en étoffe ndop, torse nu, liane de ndor-Basella alba- en large collier autour du cou, bonnet sur la tête, matchète de guerre dans son fourreau maintenu en sautoir par un baudrier d'étoffe, fusil), se rendent à la rivière avec un drapeau et deux poulets. Ils tirent deux coups de feu vers l'extérieur de la chefferie, sacrifient les deux poulets au-dessus de la rivière, dont le courant emporte le sang vers l'aval, à l'extérieur de la chefferie, « lavent » le drapeau et des lianes de ndor dans le courant, puis rôtissent sommairement et mangent les deux poulets.

Cela fait, ils reviennent au palais du chef. À proximité du palais, ils se mettent à chanter et à danser (photo 1). Le chef les attend, debout sur le podium de pierre qui domine le champ de danse. Ils alignent leurs fusils les uns contre les autres devant le chef et placent leur long tambour à membrane contre le dernier fusil. Leur leader traite les fusils, successivement, a) avec une écorce de mekam (Erythrophlaeum guineense) qui contient de l'acide prussique mortel lorsqu'on l'absorbe en dose suffisante, dont il effleure toutes les crosses puis tous les canons de fusils en en faisant le tour; $b$ ) avec un 
poulet dont il arrache la partie inférieure du bec et dont il fait couler le sang sur les fusils suivant le même parcours (photo 2); c) avec des plumes arrachées au poulet toujours selon le même parcours; d) avec de la poudre de padouk, qui est un fard rouge très utilisé dans la région. Il ponctue son action d'imprécations contre des ennemis imaginaires et d'appels à la mobilisation. Lorsqu'il a fini, chacun reprend son fusil et retourne chez soi.

Je me pose plusieurs questions : quel est l'effet de ces actions sur les fusils? Pour les intéressés, ces actions "rechargent» le fer des armes d'une capacité à crever les enveloppes ennemies et à tuer l'adversaire. Mais c'est moi qui le dis. Leur propre verbalisation, c'est : « Nos ancêtres ont toujours fait ainsi ». C'est une action efficace sur la matière. C'est une technique. Pour l'ethnologue naïf (pas vous et moi, bien entendu!), cette action ne possède aucune efficacité sur les fusils, donc c'est du « rituel » et du "symbolique " (selon le bon adage ironiquement formulé par Nigel Barley: "This is crazy, therefore it must be symbolism»). Deuxième question: quel est l'effet de ces actions sur les sujets? C'est, à mon avis, une question pertinente dans ce contexte. Probablement la seule. Mais la réponse n'est pas simple. Les effets sont réels et complexes. Les sujets ne sont plus exactement les mêmes après et avant ces actions. Il y a une "action efficace sur les sujets». Il y a une «technique de soi » qui vise le sujet-et-son-fusil avec lequel il «fait corps» dans le combat, ou faisait corps dans le passé.

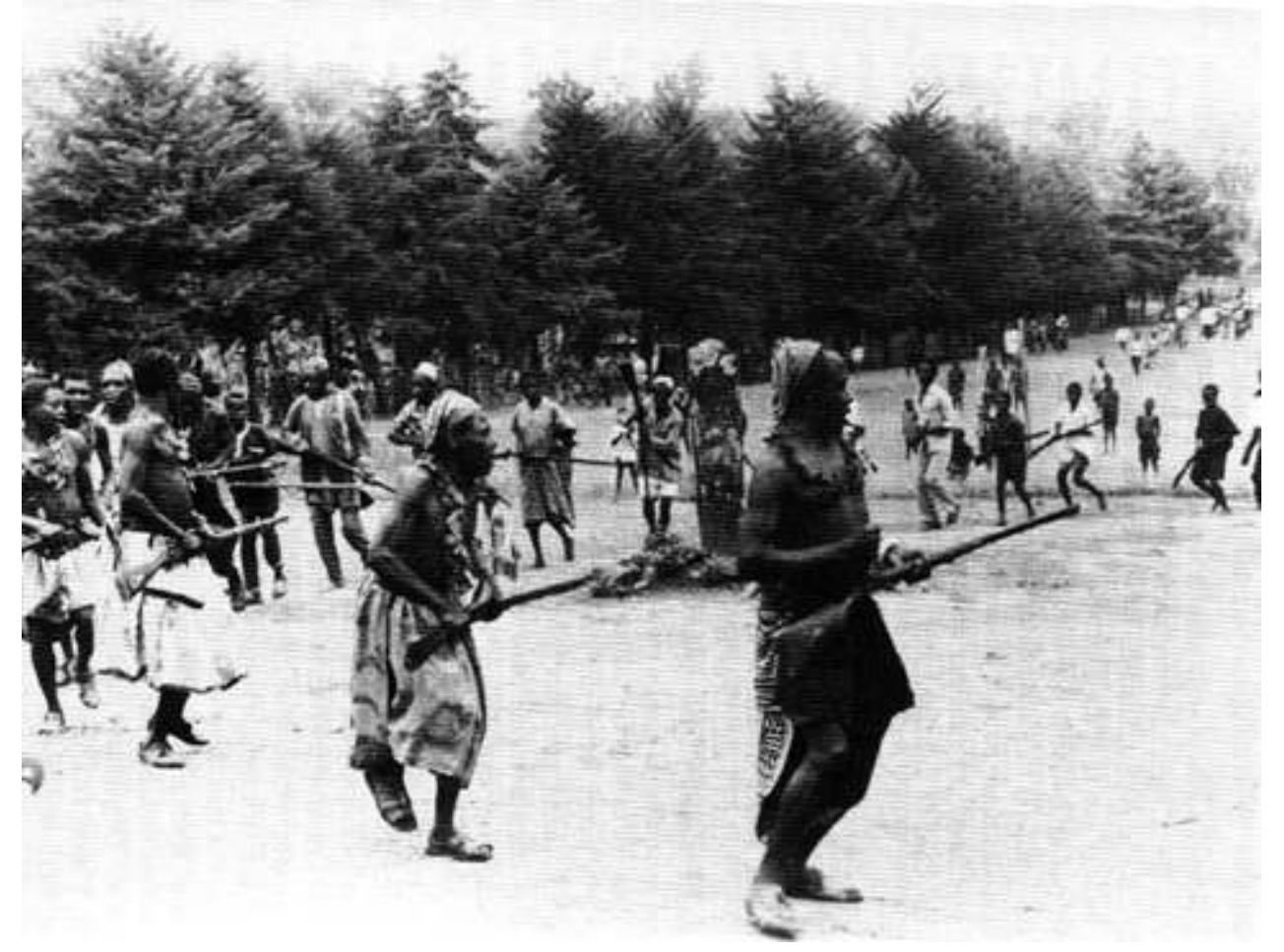

Photo 1. Le retour des guerriers le jour de Mesongong (Mankon, 1972) 


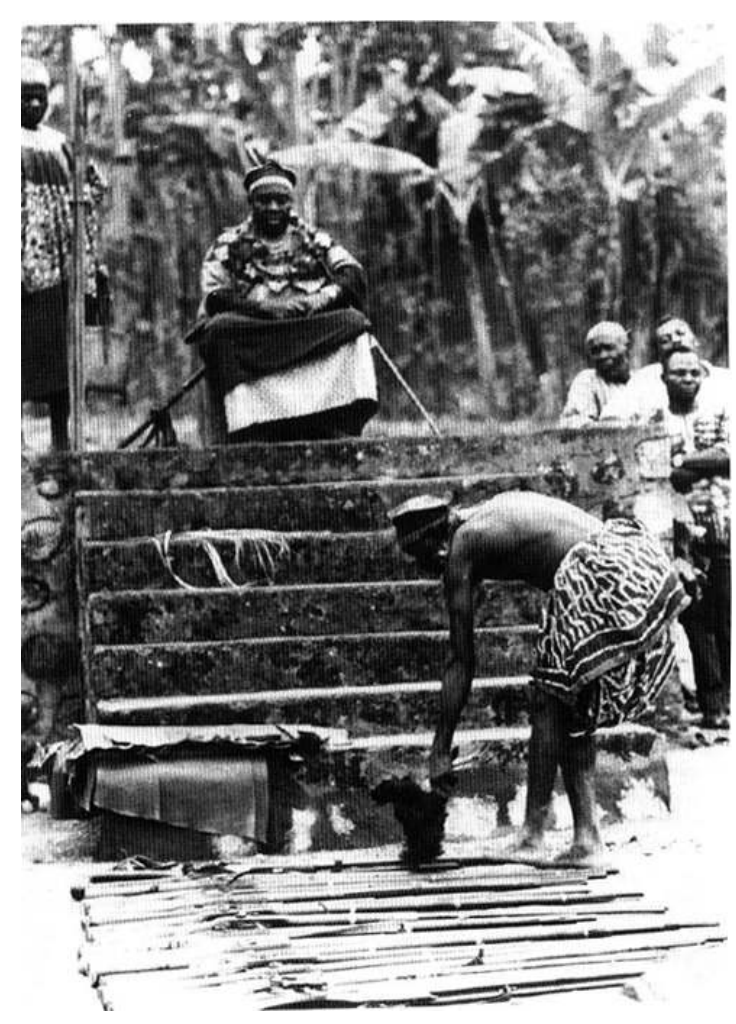

Photo 2. Le leader de la loge mesongong répand le sang d'un poulet sur les fusils accents différents, à savoir que ce qui se joue dans ces conduites, ce n'est pas seulement la communication entre sujets au moyen d'objets qui font signe dans un système codé (pace Barthes et Baudrillard), c'est une intériorisation qui permet de construire le sujet en tant que sujet de ses propres actions guerrières, et de ses propres conduites sensoriaffectivo-motrices en situation de combat plus ou moins imaginaire. Ces actions accomplissent ce qu'aucun discours, aucune parole, ne peut accomplir. La fonction performative du langage n'a en effet qu'une portée limitée. Si, dans le contexte approprié, l'énoncé «Je vous déclare mari et femme » accomplit effectivement ce qu'il énonce, il s'en faut de beaucoup qu'on puisse faire un guerrier en le déclarant guerrier. Mais, mutatis mutandis, il en est de même du mariage, et l'énoncé de M. le maire n'est rien si l'action ne précède ou ne suit pas. Il faut que le guerrier «se fasse » par des techniques de soi proposées ou imposées par la société, à la suite de quoi on peut, verbalement, le déclarer guerrier.

La parade

Techniques \& Culture, 39 | 2002 
31 Le lendemain de Njwi mesongong, a lieu Ale'ete ( "le défilé » ou « la parade »), ouvert à tous les citoyens de la chefferie de Mankon. Chacun se présente en attirail de guerre. Les femmes accompagnent les hommes. Tous et toutes se placent en fer à cheval autour du champ de danse, le podium du chef se situant dans l'ouverture du fer à cheval. Les hommes forment une ligne derrière laquelle se tiennent les femmes. En milieu d'aprèsmidi, le chef sort de son palais, escorté par ses notables, ses serviteurs et un petit orchestre. Ils arrivent en dansant d'un pas retenu. Le chef monte sur le podium. L'orchestre se met en place et la danse commence. Le chef salue. La foule répond par des salves de mousqueterie tirées en l'air (photo 3). Le chef tire quelques coups de carabine. Les guerriers, par petits groupes de deux à cinq, montent vers le podium. Ils font des bonds grotesques, miment la progression et les feintes du combat, roulent des yeux, basculent la tête en arrière en se mordant les lèvres (photo 4). Ils se redressent et s'accroupissent tour à tour. Leurs gestes sont coordonnés dans ce que Parlebas nomme de la "co-motricité ", à quoi se superpose de la "socio-motricité ", c'est-à-dire des séries d'actes accomplies en interaction motrice essentielle avec les autres sujets et avec le chef, lequel suit leur progression dès lors que le groupe de guerriers qui monte vers lui est suffisamment proche. Il réagit à leurs mimiques, brandit son javelot et le fait vibrer en en secouant la hampe. Chaque sujet, chaque groupe, est en compétition avec les autres pour accomplir la performance la plus spectaculaire.

Il ne s'agit pas à proprement parler d'un entraînement au combat. La notion et la pratique de l'entraînement étaient inconnues. C'est un quasi-jeu (non pas « du corps »), mais une conduite sensori-affectivo-motrice qui met en scène la menace, l'intimidation, la colère, les feintes du combat en face-à-face, à portée de javelot. Le mousquet, long à charger, peu fiable, relativement inefficace sauf dans un tir en batterie sous la protection des armes blanches, n'avait pas fondamentalement modifié les tactiques au XIXe siècle. Par contre, il possédait un puissant effet de mobilisation sensorielle : le bruit, la déflagration et l'émission d'un nuage de fumée brutalement expulsé après avoir été contenu dans un si petit espace, et chargé de poudres maléfiques (poussière de cœur humain desséché, écorce de mekam) permettaient au guerrier d'intérioriser la violence guerrière et sa projection à l'extérieur (photo 3). C'était et c'est toujours un instrument efficace d'introjection et d'identification.

33 Mais à l'inverse, et à la lumière de ces quasi-jeux identificateurs, il y a lieu de se demander si le parcours du combattant des armées modernes ne possède pas une dimension forte de subjectivation et d'identification. Certes, pour se livrer à une action de commando, mieux vaut être parfaitement entraîné à une marche de $50 \mathrm{~km}$ en terrain accidenté, avec $35 \mathrm{~kg}$ d'équipement, et être capable de franchir murs et barbelés. Mais cette vue technicienne est insuffisante. Le guerrier est un sujet, et c'est dans ses peurs, sa détermination à se battre, ses perceptions et ses conduites motrices routinisées qu'il faut le rejoindre. Certains aspects de l'entraînement sont d'une efficacité discutable au regard de la situation de combat. Ils sont «crazy» au sens de N. Barley, d'où je ne conclus pas qu'il sont du "symbolique » en ce qu'ils feraient signe, mais qu'ils constituent d'efficaces techniques de subjectivation identificatrice (tout en faisant effectivement signe). Exemple : "rectifier la position » en un geste brusque, sonore et mécanique, saluer, et débiter de manière non moins brusque, sonore et mécanique: "Caporal Dupont, 6ème régiment, 2ème compagnie, à vos ordres mon Colonel !». Puissante technique d'assujettissement et de production d'un sujet guerrier. 


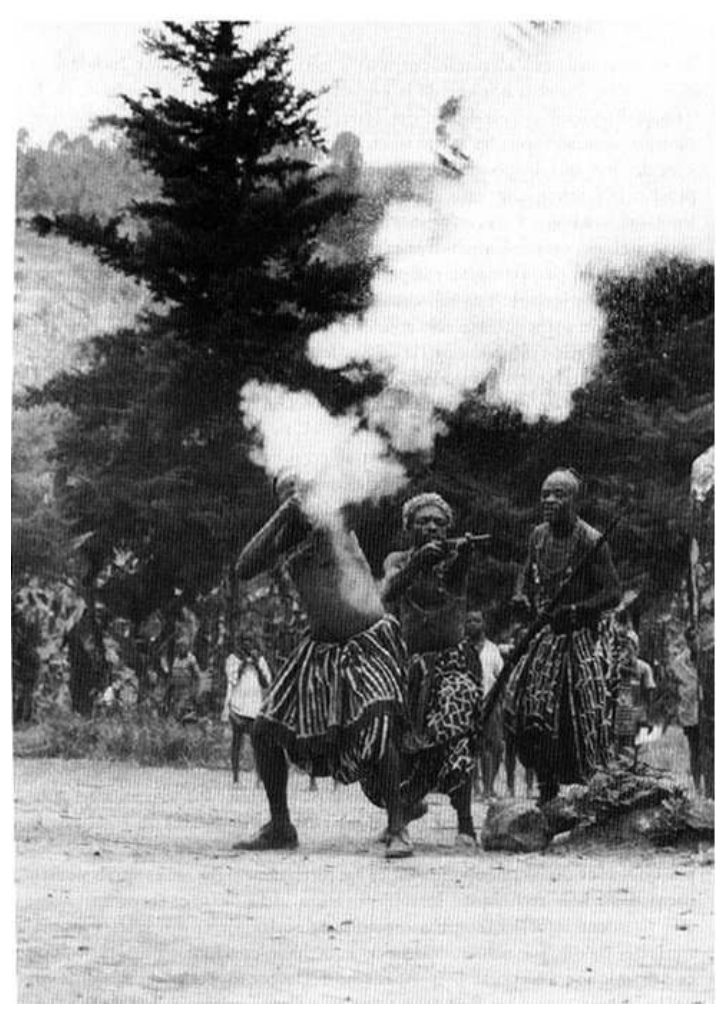

Photo 3. Salve de mousqueterie le jour de la « parade »

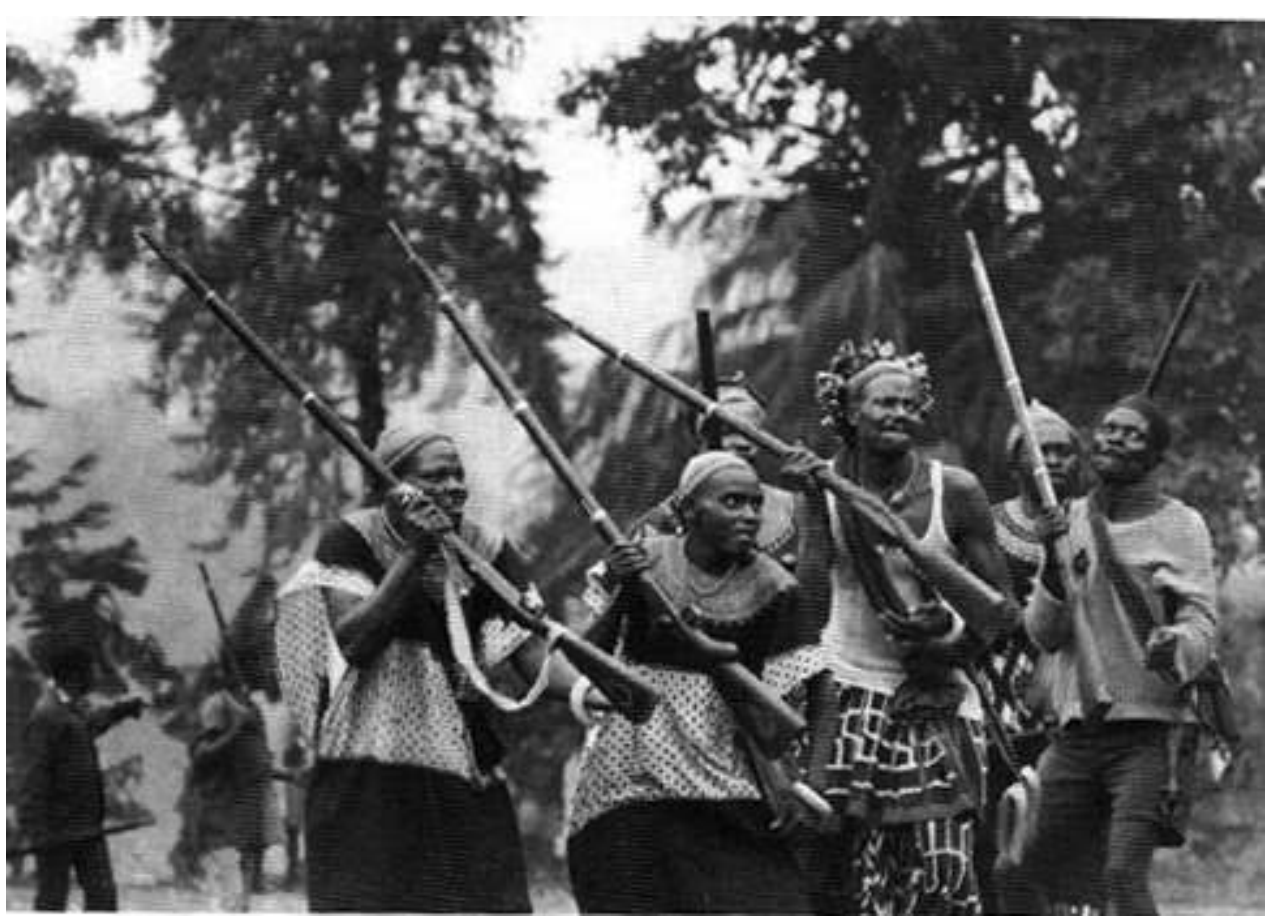

Photo 4. Les conduites sensori-affectivo-motrices du combat

L'espion

34 Le gwe (espion, homme de sac et de corde du chef). C'est le lendemain de la "parade ». Tout le monde danse autour du chef en habits de fête, au son de l'orchestre de flûtes et du chœur des filles du palais. L'ethnologue danse avec les autres, tout en se 
préoccupant de son métier d'observateur. Il a un appareil reflex dans son sac. Les mouvements de la ligne des danseurs l'amènent près de l'enclos du drapeau, une sorte de mini-camp de guerre situé en haut du champ de danse. Un homme veille à l'entrée, en position accroupie, observant les danseurs. Il repère l'ethnologue et fixe sur lui un regard inquisiteur. Pour ne pas être en reste, je sors mon appareil de photo et cadre le veilleur. C'est un gwe (sg. : espion, garde) qui s'appelle Nde' Medze. De sa posture de repos, il passe à une posture plus tonique, en tension accroupie, les phalanges de la main gauche en appui sur le sol. Il penche la tête en avant, me fixant droit dans l'objectif par dessous ses sourcils. Puis il incline la tête sur l'épaule droite, élève la main droite à hauteur des yeux. La main et le bras sont souples, l'index légèrement tendu vers l'objectif de l'appareil, le visage sérieux. Il agite sa main d'un tremblement et commence à sourire. Le sourire est ambigu. Il trahit des intentions, mais sans en laisser deviner la nature. Je commence à me sentir mal à l'aise et déstabilisé. Je fais plusieurs clichés, mais bute de plus en plus sur le maniement de l'appareil tout en essayant d'improviser au moyen de cet objet un scénario qui réponde à celui de Nde' Medze. Je l'incline lentement à droite, comme si, ce faisant, j'accomplissais une action efficace sur celui qui, dans le jeu, se pose comme partenaire/adversaire. J'étais déjà jambes fléchies pour me situer à une bonne hauteur pour les prises de vue. J'en profite pour esquisser des bonds latéraux d'esquive. Nde' Medze se prend au jeu qui tourne à une lutte d'intimidation entre l'objectif - ma seule arme - et les mimiques mille fois rodées de l'espion qui n'en est pas à sa première interaction de ce genre. La saynète se solde par un éclat de rire et des félicitations.

Le même jour, un autre gwe se promène avec un masque à gaz sur le visage, retourné sens dessus dessous, avec un tuyau d'alimentation en air pur dirigé vers le haut, par dessus sa tête. Il est couvert de petits sacs et de cornes d'antilopes bourrées de substances diverses. Il tient une trique à la main et se précipite sans crier gare sur les danseurs mal coordonnés ou sur la ligne des danseurs, à mesure qu'elle se déforme et empiète sur l'espace central du champ de danse. Il provoque des débandades. Des enfants hurlent de terreur. Là encore, il y a construction du sujet, action sur les actions des autres et assujettissement par des conduites sensori-affectivo-motrices non verbalisées, et dont la verbalisation n'aurait aucune pertinence au regard de l'effet, et guère de valeur informative.

D'autres quasi-jeux

Cette ethnographie succincte pourrait se prolonger par un panorama complet de tous les quasi-jeux en rapport avec la guerre, comme les réunions des loges de guerriers qu'on appelle mandzong, les réunions d'associations de spécialistes des médecines de guerre qui se préparaient à faire la pluie et le beau temps sur le champ de bataille, à transporter les combattants dans les airs, à incendier les maisons à distance, etc., ou encore les pratiques des fondeurs et forgerons travaillant un métal impliqué dans l'art de la guerre. Une telle entreprise dépasse le cadre de ce court article, mais on en trouvera des éléments dans deux publications (Bayart \& Warnier s/presse; Warnier, à paraître). Dans tous ces exemples ethnographiques, on constate, sans exception, la présence de conduites sensori-affectivo-motrices institutionnalisées, étayées dans et par une culture matérielle elle-même objet d'institutionnalisation, confinant dans certains cas à la technologie (pour la forge par exemple) ou à ce que je répugne à appeler le «rituel» (dans la médication des fusils par exemple), et impliquant toutes, sans exception, la production de sujets guerriers dans leurs algorithmes moteurs, leurs 
identifications, leur engagement affectif et leur assujettissement à une souveraineté. C'est la catégorie du «jeu», au sens où Winnicott en parlait, qui me semble la plus adéquate pour englober ces phénomènes, à savoir ce que fait un sujet dans un espace transitionnel, et qui est de l'ordre de la création de soi et de son monde.

Qu'est-ce que ça leur fait?

Mon iconoclasme vise les différents paradigmes de la technologie culturelle qui définit la technique comme une action efficace sur la matière. Les acquis de ces paradigmes sont immenses. Ils ont permis d'établir fermement les techniques comme un objet légitime dans le champ de l'anthropologie, et comme élément essentiel de toute civilisation. Ils ont mis le geste et les dynamiques au centre de leurs préoccupations. Une masse impressionnante de techniques d'une simplicité apparente, d'une grande complexité du point de vue des chaînes opératoires et des contraintes techniques, ont été décrites et analysées. Mais il me semble que ces paradigmes s'essoufflent, car il n'ont pas pris en compte ce qui se passait, chemin faisant, dans d'autres domaines du savoir autour de la question du sujet. Contextualiser la technique dans la ou les cultures, bien que nécessaire, est devenu insuffisant si le prix à payer est le maintien d'une coupure plus ou moins tranchée entre un acteur technique réduit au « corps » et la matière ouvrée; si l'action est exclusivement perçue comme une action transitive; si le corps de l'acteur est réduit à l'anatomo-physiologique; si le sujet est absent de l'analyse.

En d'autres termes, il n'existe aucune technique d'objet qui n'engage que « le corps ». Il n'existe aucune technique d'objet qui n'engage les conduites sensori-affectivo-motrices du sujet. La question de la chaîne opératoire -à savoir : qu'est-ce que cela fait à la matière et comment ?- se double toujours d'une autre question d'orientation inverse : qu'est-ce que cela fait au sujet et comment ? Il n'existe aucune technique d'objet qui ne soit en même temps une technique de soi. Le travailleur -la technologie culturelle en a fourni maints exemples sans pour autant thématiser cet aspect des choses- n'est pas une machine. Dans le travail, l'obstacle (l'objet) n'est pas seulement la planche de bois ou le fer à forger. C'est le travailleur lui-même dans la mesure où l'efficacité de son action dépend de la mise au point de ses propres algorithmes moteurs efficaces, qu'il travaille et rectifie sans cesse, et qui sont articulés à l'engagement des sens et de l'affectivité. Mais cet objet n'en est pas un: c'est un sujet. L'action efficace sur la matière rebondit en une action efficace de soi sur soi. Dire qu'elle est l'action d'un corps-moteur sur une matière-mue ne prend pas en compte cet aspect très particulier de l'action d'un organisme qui est un sujet.

A fortiori s'il s'agit des sports et des jeux dits «du corps». La transposition à ces conduites ludo-motrices du schéma du "corps-moteur d'un corps-mû » est pour le coup erronée. J'ai tenté de montrer, par des exemples ethnogra-phiques, que, si production il y a, ce n'est pas d'un corps qui serait objet de production, mais d'un sujet, sous le quadruple aspect de l'avènement du sujet (devenir quelqu'un), de ses identifications, de son assujettissement à des réseaux institutionnalisés d'actions sur les actions des autres (Foucault disait des "gouvernementalités»), et d'intégration d'algorithmes sensori-moteurs. C'est cette institutionnalisation (par exemple dans les pratiques de guerre et l'organisation en loges de guerriers), cet élément de collectivité, de récurrence et de prévisibilité, qui fait que les techniques de soi et d'identification dépassent la préoccupation "d'être soi » à titre purement individuel, et par conséquent de peu d'intérêt pour l'anthropologue. 


\section{L'effet Magritte}

d'opérer le passage maussien et schilderien entre le corps et le sujet. Pour cela, il faut en parallèle mener un travail de déblayage de tous les poncifs concernant «le corps ", dont on trouve un compendium dans les ouvrages de D. Le Breton. À la différence de cet auteur, je ne pense pas que les sociétés occidentales aient oublié le corps ou l'aient voué aux gémonies. Toutes les sociétés oublient le corps, pour la simple raison que le corps a vocation à se faire oublier sauf dans ses dysfonctionnements. En effet, c'est le lieu d'un double inconscient: l'inconscient comme refoulé au sens freudien, et l'inconscient moteur au sens de Parlebas (1999: 171) :

«Si le gardien de but de football qui "sort" sur un "centre" de l'ailier adverse ou le barreur qui vire de bord devaient "prendre conscience" de tous les éléments de la situation motrice qui sont à la source de leur conduite, ils seraient condamnés à rester figés sur place devant l'ampleur de ces exigences .»

L'inconscient moteur (dans lequel Parlebas inclut le sens freudien) est libé-rateur. Il joue un rôle prodigieux d'économie, dans toutes les sociétés, y compris celles de la tradition.

À la différence de D. Le Breton et de Csordas (1994), je ne pense pas qu'on puisse espérer quoi que ce soit d'une anthropologie phénoménologique accomplissant la « réduction au corps " car une telle anthropologie vise directement le «sens » et la communication, en faisant l'économie d'une boîte à outils permettant de pratiquer une ethnographie des conduites motrices telle qu'on la trouve très précisément dans la praxéologie motrice de Parlebas. En d'autres termes, l'anthropologie phénoménologique est victime de ce que j'appelle «l'effet Magritte»: elle parle du tableau, de la représentation de la pipe, et non de la pipe. Sur le tableau représentant une pipe, Magritte, en surréaliste et donc en réaliste conséquent, a écrit : «ceci n'est pas une pipe ». Il a raison : on ne peut pas bourrer du tabac dans le tableau, ni l'allumer, ni le fumer.

En dernier lieu, et contrairement à D. Le Breton, je pense que ce qui est pertinent, ce n'est pas «le corps", mais les conduites sensori-affectivo-motrices étayées en culture matérielle.

\section{BIBLIOGRAPHIE}

Bayart, Jean-François \& Jean-Pierre Warnier

$\mathrm{s} /$ presse Culture matérielle et subjectivation politique. Paris : CERI.

Csordas, Thomas

1994 Embodiment and Experience. The Existencial Ground of Culture and Self. Cambridge : Cambridge

University Press.

Julien, Marie-Pierre \& Jean-Pierre Warnier

1999 Approches de la culture matérielle. Corps à corps avec l'objet. Paris : L'Harmattan.

Techniques \& Culture, 39 | 2002 
Karsenti, Bruno

1997 L'Homme total. Sociologie, anthropologie et philosophie chez Marcel Mauss. Paris : Presses

Universitaires de France.

Mauss, Marcel

1950 « Les techniques du corps », pp. 363-386, in Cl. Lévi-Strauss (ed.), Sociologie et anthropologie.

Paris : Presses Universitaires de France (1ère édition 1936, Journal de Psychologie, XXXII, 3-4).

Mendel, Gérard

1998 L'Acte est une aventure. Du sujet métaphysique au sujet de l'acte-pouvoir. Paris : La Découverte.

Parlebas, Pierre

1999 Jeux, sports et sociétés. Lexique de praxéologie motrice. Paris : INSEP.

Schilder, Paul

1923 Das Körperschema. Ein Beitrag zur Lehre vom Bewusstzein des eigenen Körpers. Berlin : J. Springer. 1935 The Image and Appearance of the Human Body: Studies in the Constructive Energy of the Psyche.

London : Kegan Paul.

Tisseron, Serge

1999 Comment l'esprit vient aux objets. Paris : Aubier.

2000 Petites mythologie d'aujourd'hui. Paris : Aubier.

Warnier, Jean-Pierre

1985 Échanges, développement et hiérarchies dans le Bamenda pré-colonial - Cameroun. Stuttgart : Franz Steiner Verlag Wiesebaden.

1999 Construire la culture matérielle. L'homme qui pensait avec ses doigts. Paris : Presses Universitaires de France.

2001 « A praxeological approach to subjectivation in a material world », Journal of Material Culture $6(1): 5-24$.

À paraitre Le roi-peau des Grassfields du Cameroun.

\section{RÉSUMÉS}

Les techniques du corps peuvent-elles être considérées comme des actions efficaces du corpsmoteur sur le corps-matière d'œuvre ? Le présent article conclut par la négative à partir d'une analyse des quasi-jeux guerriers pratiqués dans les chefferies du Cameroun de l'ouest. En s'engageant dans des conduites sensori-affectivo-motrices étayées sur la culture matérielle de la guerre, les hommes mettent en œuvre des techniques de soi qui contribuent à les identifier de manière efficace. En d'autres termes, la prise en compte du «sujet " plutôt que du "corps " permet de poser la question de l'action efficace sur le sujet qui double les pratiques d'action efficace (ou inefficace) sur la matière - que ce soit celle du corps ou des objets.

\section{Warrior games in West Cameroun. Some iconoclast remarks}

Can body techniques be considered as efficient action of the body as a muscular motor on the body as matter ? After analysing the warrior games of West Cameroon chiefdoms, this paper gives a negative answer to the question. While engaging in sensori-affectivo-motory conducts baked on the material culture of war, the men bring into play techniques of the self which contribute to identify them efficaciously. In other words, the taking into account the "subject » rather than the " object " (the body) enables to attribute the efficient action to the subject who furthermore, practises efficient (or inefficient) action on matter, may it be that of the body or of some other object. 
Los juegos guerreros del Oeste de Camerún. Algunas propuestas iconoclastas.

Se pueden considerar a la técnica del cuerpo como acciones eficaces del cuerpo-motor sobre el «cuerpo-materia de obra »? El presente artículo responde a esta pregunta con una negativa, a partir de un análisis de los cuasi-juegos guerreros, que se practican en las jefaturas del Oeste de Camerún. Implicándose en conductas sensoriales-afectivas-motrices que se apoyan en la cultura material de la guerra, los hombres ponen en práctica técnicas que contribuyen a identificarles de manera eficaz. Dicho en otros términos, el hecho de tomar en cuenta el «sujeto » antes que el «cuerpo » permite plantear la acción eficaz sobre el sujeto que repite las prácticas de acción eficaz (o ineficaz) sobre la materia (sea esta la materia del cuerpo o la de los objetos).

INDEX

Mots-clés : corps, jeux, Cameroun, conduites motrices, guerre, identification, sujet

\section{AUTEUR}

\section{JEAN-PIERRE WARNIER}

Université René Descartes -Paris V, Faculté des Sciences humaines et sociales. 12 rue Cujas, 75005 Paris 